\title{
Quasi-dynamic model of the energy efficiency degradation coefficient for an air-to-water heat pump
}

\author{
Krzysztof Piechurski ${ }^{1, *}$, Małgorzata Szulgowska-Zgrzywa ${ }^{1}$, and Jan Danielewicz ${ }^{1}$ \\ ${ }^{1}$ Wrocław University of Science and Technology, Wybrzeże Stanisława Wyspiańskiego 27, \\ 50-370 Wrocław, Poland
}

\begin{abstract}
Operating in part-load conditions significantly affects the energy efficiency of the air-to-water heat pumps. The currently used method of testing the devices does not provide the necessary data for estimating the degradation of the device's efficiency in part-load operating mode. The value of the degradation coefficient $C c=0.9$ adopted in accordance with the EN 14825 standard is generally used. However, this value often does not reflect the actual degree of the COP reduction. Hence the new quasidynamic model and the calculation approach to the degradation coefficient were proposed. The model reflects the real and overall reduction between the full and part-load COP, not only the energy consumption in the standby mode. The estimation was made using the field measurement data of the air-to-water heat pump supplying heat to the low temperature installation. It has been proved that the determination of the main parameter is possible using a relatively small amount of data without losing an accuracy.
\end{abstract}

\section{Introduction}

Air-to-water heat pumps are more and more frequently used as an independent source of heat in low-energy buildings. In order to obtain the highest energy efficiency, it is necessary to analyze the cooperation of the heat source and the heating installation. In such analyzes, operation of the heat pump with partial load is often neglected. However, it turns out to have a significant impact on the COP and therefore also on SCOP. Due to the nature of the air-towater heat pumps, for a great part of the heating season (for temperatures above the bivalent point) it is necessary to reduce the generated heat power. For fixed-capacity units the only possibility is to switch the compressor on and off repeatedly. In most of the new devices, the capacity control based on changing the rotational speed of the compressor by a frequency inverter is commonly used. However, this kind of regulation is limited to the point of maximum power reduction. Below this point, the device will additionally start cycling switching the compressor on and off. Furthermore, considering these reductions may affect the correct sizing of the heating unit. Unfortunately, the currently used method of testing the devices in accordance with the EN 14511 [1] standard does not provide the necessary data for estimating the degradation of the device's efficiency connected with these factors. Tests

${ }^{*}$ Corresponding author: krzysztof.piechurski@.pwr.edu.pl 
are performed for full capacity and uninterrupted operation of the device in the steady-state mode. This results in significant differences between the manufacturer and the actual COP values, which for the device tested are shown in Fig. 1. Proper compatibility in the area of power adjustment and large discrepancies in the area where the power of the heat pump is much higher than the heating demand of the building is noticeable.

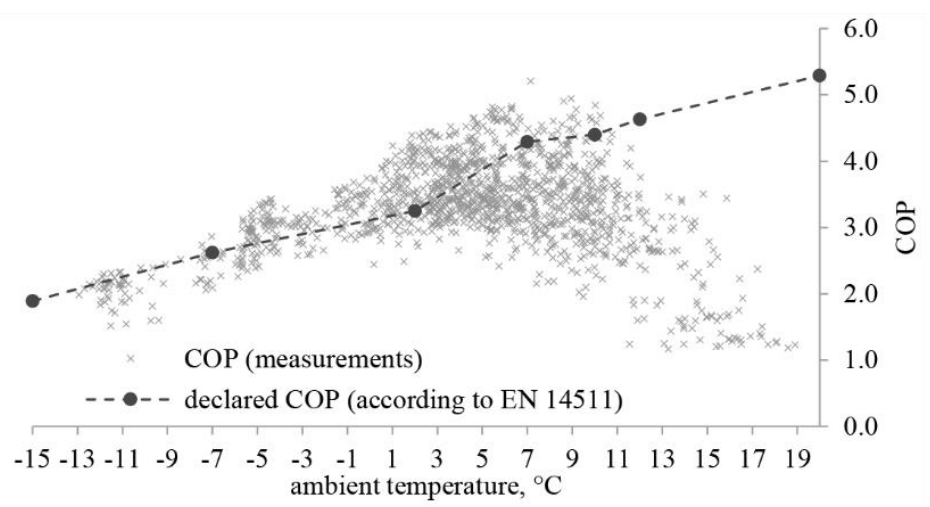

Fig. 1. The manufacturer COP data compared with the sample measured data.

In previous works [2], the authors have already discussed the problem of inaccuracies resulting from the imprecise determination of data for the calculation of the reduction coefficient. These works has focused on possible errors related to inaccurate input data for SCOP analyzes. This publication systematizes the literature research connected to the COP reduction as a result of the part-load operation. A quasi-dynamic model and method for calculating the operational value of the degradation coefficient has been proposed.

\section{The degradation coefficient in European standards and the literature}

The method of calculating the COP reduction as a result of the part-load operation of an airto-water heat pump is given i.a. in EN 14825 [3] standard. The tests are performed at several measuring points in variable heat load conditions. One of the data calculated is the degradation coefficient $(C c)$, which is defined as the energy consumed by the device when the compressor is switched off. It should be determined for each part-load ratio according to the equation (1).

$$
C c=1-\frac{\text { measured power of compressor off state }}{\text { total power input (full capacity at the part load conditions) }}
$$

The standard also suggests to assume the value of $C c$ equal to 0.9 in case the test was not performed. The value is then used to calculate the reduction of declared COP $(C O P d)$ under the same temperature conditions as for the test under part load conditions according to the equation (2). The reduced coefficient of performance is called part-load COP $\left(C O P_{P L}\right)$.

$$
C O P_{P L}=C O P_{d} \cdot \frac{P L R}{C c \cdot P L R+(1-C C)}
$$

A similar approach is described in the standard EN 15316 [4]. The energy consumed by the device in the stand-by mode $\left(Q_{\text {el.stand-by }}\right)$ is calculated on the basis of the power determined by the manufacturer and the estimated working time in this mode. The time, however, 
changes in accordance with the heating demand of the building $\left(Q_{H}\right)$ and the heat pump's power. The amount of the energy consumed is then included when calculating the reduced COP of the device in accordance with equation (3).

$$
\operatorname{COP}_{P L}=\frac{Q_{H}}{\frac{Q_{H}}{C O P_{d}}+Q_{e l . s t a n d-b y}}
$$

Although both methods base on energy consumed in the stand-by mode, the reduced COP obtained on their basis often differ significantly and show great discrepancies in relation to the measured data $[2,5]$. E. Fuentes et al. [5], on the basis of experimental research, have observed that start-up losses have significant impact on the efficiency of the device. However, this is neglected in calculations according to the current European standards (e.g. EN 14825) for air-to-water, water-to-water and brine-to-water systems. In order to take this effect into account, authors [5] proposed an improved equation (4) to define the COP reduction factor $(P L F)$, adding the degradation coefficient $C d$ as defined in EN 14825 for airto-air and water-to-air heat pumps. They proved that the combination of these parameters could improve the accuracy of the part-load COP estimation.

$$
P L F=\frac{1}{1+\frac{C d(1-P L R)}{1-C d(1-P L R)}+(1-C c) \frac{1-P L R}{P L R}}
$$

The Italian standard UNI 10963 [6], on the other hand, proposes a statistical determination of two parameters ( $a$ and $b$ ) based on the measurement data from the short test of the device. The advantage of the method is that it is sufficient to test in a single experiment both at the part-load and the full-load. Consideration of the device's efficiency ratio both at full and partial load increases the accuracy of the results. The COP reduction factor $(P L F)$ is then calculated according to equation (5). Similarity to the equation (2) is noticeable, where parameter $a$ corresponds to the $C c$ value, and $b$ to the $(1-C c)$.

$$
P L F=\frac{P L R}{a \cdot P L R+b}
$$

The disadvantage of the method is the necessity of having a device and a suitable measuring set-up, because the current tests according to the EN 14511 standard do not provide the required data. Therefore, the change of the heat pumps testing standard seems to be necessary, considering the current methods of SCOP calculations and the technological progress in this field.

\section{Description of the research and analysis}

\subsection{Description of the real object installation}

The analyzes presented in this paper were based on measurement data of the air-to-water heat pump, supplying heat to the low temperature installation. The heat is distributed to the offices of approx. $300 \mathrm{~m}^{2}$ situated on the $1 \mathrm{st}$ floor and to the air handling unit room in which the heat source - the air-to-water heat pump with a compressor (split type) of the nominal power of $10.6 \mathrm{~kW}\left(\mathrm{~A} 2 / \mathrm{W} 35^{\circ} \mathrm{C}\right)$ - is installed. The heat pump performance data: the heating capacity $\left(Q_{H P}\right)$ and the declared coefficient of performance $(C O P d)$ measured according to EN 14511 standard are shown in Table 1. The unit works in the monoenergetic mode. In the periods of insufficient heating capacity or the ambient temperature below $-15^{\circ} \mathrm{C}$, the built-in electric heater delivers auxiliary energy. The installation does not have any heat buffer and is not used for domestic hot water. For further analysis, it is also important that the unit has 
a frequency inverter installed. It allows the capacity control of the heat pump up to the value of $5.0 \mathrm{~kW}$.

Table 1. Declared heating capacity $\left(Q_{H P}\right)$ and $C O P_{d}$ of the unit, according to EN 14511.

\begin{tabular}{|c|c|c|c|c|c|c|c|c|}
\hline & $\mathbf{T}_{\mathbf{a}},{ }^{\circ} \mathbf{C}$ & $\mathbf{- 1 5}$ & $\mathbf{- 7}$ & $\mathbf{2}$ & $\mathbf{7}$ & $\mathbf{1 0}$ & $\mathbf{1 2}$ & $\mathbf{2 0}$ \\
\hline \multirow{2}{*}{$\mathrm{QHP}_{\text {HP }}(\mathrm{kW})$} & $\mathrm{T}_{\text {in }}=35^{\circ} \mathrm{C}$ & 5.80 & 8.47 & 10.60 & 14.60 & 14.80 & 15.82 & 17.90 \\
\cline { 2 - 9 } & $\mathrm{T}_{\text {in }}=45^{\circ} \mathrm{C}$ & 5.20 & 7.50 & 10.00 & 13.10 & 14.10 & 14.70 & 16.80 \\
\hline \multirow{2}{*}{$\mathrm{COP}_{\mathrm{d}}$} & $\mathrm{T}_{\text {in }}=35^{\circ} \mathrm{C}$ & 1.89 & 2.62 & 3.25 & 4.29 & 4.40 & 4.63 & 5.29 \\
\cline { 2 - 9 } & $\mathrm{T}_{\text {in }}=45^{\circ} \mathrm{C}$ & 1.50 & 2.10 & 2.80 & 3.10 & 3.40 & 3.50 & 4.10 \\
\hline
\end{tabular}

For the purpose of this paper, the analysis of the system behavior over the heating season (October 2013 - March 2014) has been made. The flow, the inlet and return temperatures, the ambient temperature $\left(T_{a}\right)$ and the electric power consumption $\left(Q_{e l}\right)$ were measured. The data collected allowed to analyze the power of the heat pump $\left(Q_{H P}\right)$ and the energy transferred to the building $\left(Q_{B}\right)$ in each hour. The real coefficient of the performance $\left(C O P_{P L}\right)$ was calculated as the ratio of $Q_{B}$ to $Q_{e l}$. The $P L R$ value was calculated as the ratio of $Q_{B}$ to $Q_{H P}$, also for each hour. The $C O P_{d}$ values were assigned by the linear interpolation for $T_{a}$ and the inlet temperature. $P L F$ was calculated as the ratio $C O P_{P L}$ to $C O P_{d}$. The results of the calculations are presented in the further part of the article (Fig. 2).

\subsection{Data analysis method}

The aim of the tests described in the EN 14825 [3] standard is to determine the degradation coefficient $C c$ based on a short test under steady-state conditions. A similar test is proposed in standard UNI 10963 [6], but the device is tested in conditions determined in at least 2 points. The authors of this article decided on a completely different approach. The purpose of the analyzes is to determine the method of testing the heat pump under quasi-dynamic conditions. In the first stage of the research, the parameters were estimated using the statistical analysis of the operating data. The measurements were recorded in 1 minute intervals and an hourly step was used for calculations.

As the analyzed relationship between PLR and PLF is not linear, a nonlinear estimation method was performed. The nonlinear estimation module in the Statistica package that allows to define any regression model was used. The method of least squares was used for data fitting. For the purposes of minimization using the least squares method the LevenbergMarquardt method was used, as proposed in the Statistica for such tasks.

The evaluation of the model fitting was performed by checking the proportion of the dependent variable translated by the model. This parameter can be considered as an equivalent to $\mathrm{R}^{2}$ (coefficient of determination) evaluated when analyzing, for example, multiple regression. What is important, even if the values of the dependent variable do not follow the normal distribution, this parameter allows to assess how well the model fits the data. The normality plots were also analyzed. The evaluation of the plots was used to exclude outliers and additional selection of measurement data.

In order to check the repeatability of the parameters estimated on the basis of measurements, the data were divided into sets covering a similar working time of the device. On this basis, it was determined whether the proposed statistical method is efficient. In earlier works, significant differences in the COP characteristics in analyzed months of the installation's operation were demonstrated. Hence, in this article those data were divided into data packets $(1-6)$ in the following months. As a result of the preliminary data analysis, some cases were selected to reject outliers and erroneous measurement data. To minimize the number of data points adversely impacting the results obtained, it was also decided to narrow the analyzed range of parameters. In the case of work with a partial load, for the correct 
determination of the parameters of the function, the key range of the temperature of the lower source varied from $0^{\circ} \mathrm{C}$ to $10^{\circ} \mathrm{C}$ and the $P L R$ from 0.17 to 0.80 .

\section{The quasi-dynamic model of the energy efficiency degradation coefficient}

\subsection{The assumptions for the model}

The first step of the analysis was to determine the operational degradation coefficient $C c$, based on the model proposed by many authors and the EN 14825 standard (6). The aim was to confront the obtained value with the values recommended by the standard and the literature.

$$
P L F=\frac{P L R}{C c \cdot P L R+(1-C c)}
$$

It should be emphasized that in case of the analysis performed, the obtained value of the $C c$ coefficient is not strictly consistent with its definition in the EN 14825 standard. By determining this parameter statistically on the basis of the measured PLR and PLF values, the obtained coefficient covers all the factors that contribute to the COP reduction of the device. However, according to the test method described in EN 14825 standard, it is defined only as part of the energy consumed in the stand-by mode. With regards to the results, it seems to be too much a simplification. The results obtained from such a test for new devices are usually $0.96-0.99$, which is much more than the value proposed by the standard in cases where the test was not carried out (0.9).

Taking into account the results of previous work [2] and the literature $[5,7,8]$ which challenge the method described in the EN 14825 standard, a new definition and calculation approach to the degradation coefficient were proposed. The analytical approach proposed by the authors is aimed to test the devices in semi-dynamic conditions instead of single point measurements. The statistical data analysis is applied in order to determine the part-load degradation of $C O P_{P L}$ to $C O P_{d}$ tested in accordance with the standard EN 14511. The authors assume that it should rather reflect the real and overall reduction between the full and partload COP, not only the energy consumption in the stand-by mode. The dependence of the $P L F$ value to the $P L R$ shows a logarithmic relation (the COP of the device significantly decreases with a large power disproportion), hence a logarithmic function was proposed. In order to avoid generating negative values, it was necessary to shift the function to a point $(0,0)$. Finally, the operational degradation coefficient was defined as parameter $a$ with the logarithmic function describing the connection between PLF and PLR according to the equation (7).

$$
P L F=1+a \cdot \ln \left(P L R+e^{(-1 / a)}\right)
$$

\subsection{Results of the analysis and discussion}

On the basis of the measured data sets, the statistical analysis was performed to determine one parameter, describing the degradation of the COP of the heat pump working in the partload in relation to the full-load. This parameter includes the influence of all factors lowering the COP of the device in case of work in the part-load mode. Table 2 summarizes the results of estimating the $C c$ parameter for the analyzed functions in data sets 1-6. Despite the different number and distribution of measuring points in the particular sets, a very good repeatability of results for both parameters was obtained. This is evident by the $U$ value, 
describing the percentage deviation from the average rate $\left(C c_{a v}\right.$ and $\left.a_{a v}\right)$ for all sets according to the equations (8) and (9) respectively for $C c$ and $a$ parameter.

$$
\begin{aligned}
& U=\left|\frac{C c-C c_{a v}}{C c_{a v}}\right| \cdot 100 \% ; \\
& U=\left|\frac{a-a_{a v}}{a_{a v}}\right| \cdot 100 \%
\end{aligned}
$$

Whereas there is a significant spread of analyzed measuring points, both functions achieve similar and satisfactory rate of the variance explained parameter $\mathrm{R}$. The obtained value of the $C c$ parameter varies from 0.79 to 0.81 , with the average of 0.80 . The average deviation from the average value is $0.8 \%$. The result parameter is much lower than the available test results carried out by the manufacturers and the value proposed by the EN 14825 standard. This seems to confirm the fact that neglecting the influence of factors other than the energy consumed in stand-by mode for COP degradation of air-to-water heat pumps operating in part load causes significant inaccuracies.

Similar accuracy of the estimated parameter was achieved for the parameter $a$ in proposed function (7). The values obtained range from 0.27 to 0.29 with the average of 0.28 . The average deviation from the average parameter value for all the sets is $1.7 \%$.

Analyzing the course of the functions (Fig. 2) it is evident that both of them well illustrate the actual connection between PLF and PLR. What is more important, the parameter estimation results are satisfactory and comparable for both sets of large and small number of cases (respectively set 5/6 and 1). The authors of this article do not negate the function proposed by EN 14825. However, the standard describes in details the definition and guidelines to determine the degradation coefficient $C c$ and the fact that the specific part-load $\mathrm{COP}$ values should be considered. For these reasons, direct use of function (6) as a quasidynamic model may generate significant interpretation problems. In the range tested, both functions (formulas 6 and 7) have a similar course, however for the other parameter values it will differ as described below and presented on Fig. 3.

\begin{tabular}{|c|c|c|c|c|c|c|c|c|}
\hline \multirow{2}{*}{\multicolumn{2}{|c|}{ Function / parameters }} & \multicolumn{6}{|c|}{ Number of data set } & \multirow{3}{*}{$\begin{array}{r}\text { Average } \\
0.80\end{array}$} \\
\hline & & \multirow{2}{*}{$\begin{array}{l}1 \\
0.80\end{array}$} & \multirow{2}{*}{$\frac{2}{0.79}$} & \multirow{2}{*}{$\begin{array}{l}3 \\
0.81\end{array}$} & \multirow{2}{*}{$\begin{array}{l}4 \\
0.80\end{array}$} & \multirow{2}{*}{$\begin{array}{l}5 \\
0.80\end{array}$} & \multirow{2}{*}{$\begin{array}{l}6 \\
0.81\end{array}$} & \\
\hline \multirow{3}{*}{$\mathrm{PLF}=\frac{1}{C c \cdot P L R+(1-C c)}$} & $\mathrm{a}$ & & & & & & & \\
\hline & $\mathrm{U}$ & $0.4 \%$ & $1.0 \%$ & $1.0 \%$ & $0.7 \%$ & $0.7 \%$ & $0.9 \%$ & $0.8 \%$ \\
\hline & $\mathrm{R}$ & 0.856 & 0.807 & 0.831 & 0.771 & 0.805 & 0.737 & 0.801 \\
\hline \multirow{3}{*}{$\mathrm{PLF}=1+a \cdot \ln \left(P L R+e^{\left(-\frac{1}{a}\right)}\right.$} & $\mathrm{Cc}$ & 0.28 & 0.28 & 0.27 & 0.28 & 0.29 & 0.28 & 0.28 \\
\hline & $\mathrm{U}$ & $1.5 \%$ & $0.8 \%$ & $4.3 \%$ & $0.7 \%$ & $3.0 \%$ & $0.3 \%$ & $1.7 \%$ \\
\hline & $\mathrm{R}$ & 0.844 & 0.808 & 0.837 & 0.781 & 0.828 & 0.731 & 0.805 \\
\hline \multicolumn{2}{|l|}{ Number of cases } & 82 & 160 & 197 & 298 & 437 & 409 & 264 \\
\hline
\end{tabular}

Table 2. Results of non-linear regression of $C c$ and $a$ parameters for the analyzed functions. 

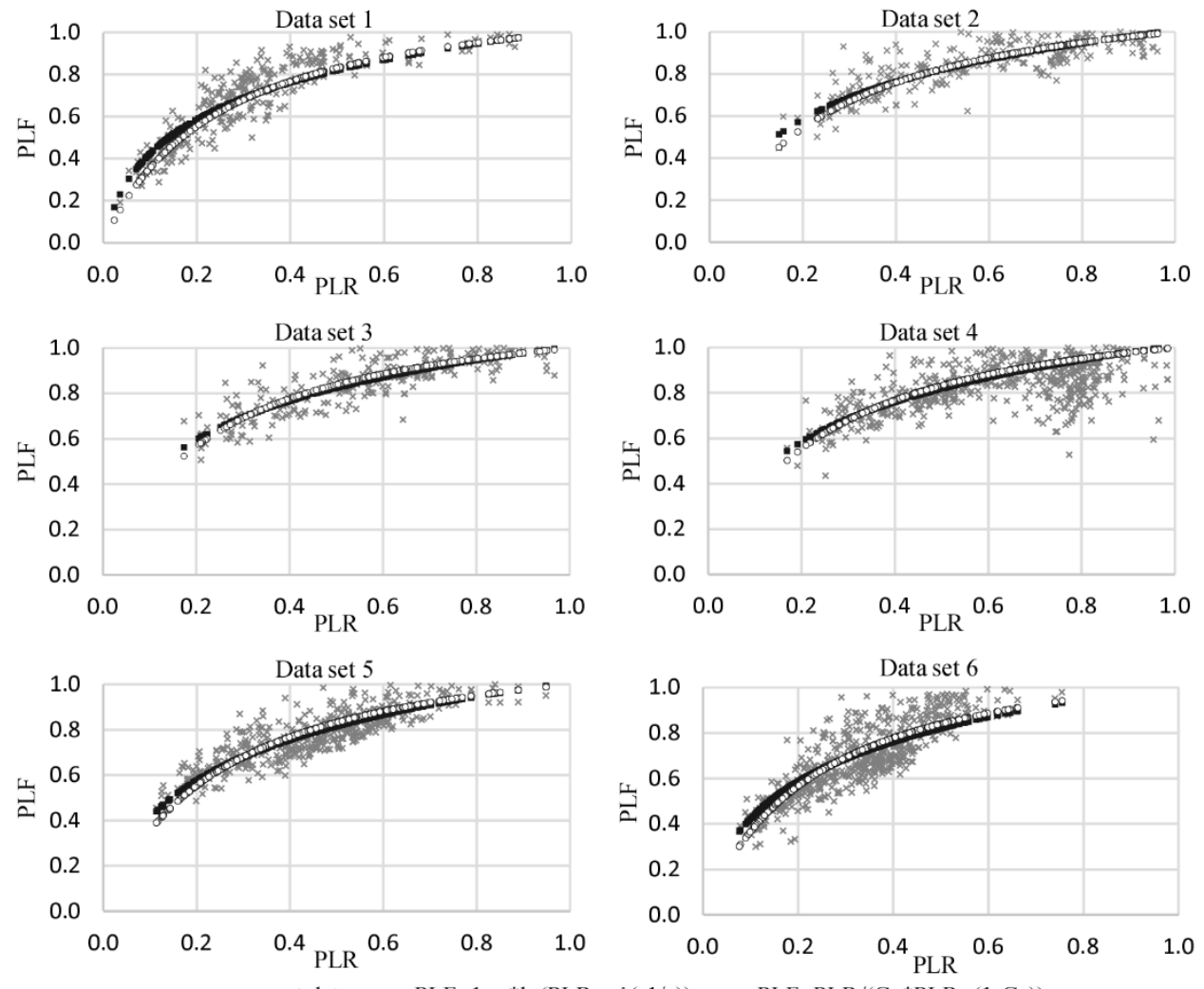

$\times$ measurement data $\quad \cdot \mathrm{PLF}=1+\mathrm{a}^{*} \ln \left(\operatorname{PLR}+\mathrm{e}^{\wedge}(-1 / \mathrm{a})\right)$

$\circ \mathrm{PLF}=\mathrm{PLR} /(\mathrm{Cc} * \mathrm{PLR}+(1-\mathrm{Cc}))$

Fig. 2. The course of the functions estimated compared with sets of measured data.

Fig. 3 presents the differences in the functions analyzed, for example for parameter values of $C c$ from 0.99 to 0.7 and $a$ between 0.4 and 0.03 . The calculated PLF differ especially for lower part-load ratio, below 0.2 . In case of the new formula (7), it may be an advantage over the (6). First of all, it reproduces the presented measurements well, but it also fits well with the data from the literature [5]. For modern heat pumps the function proposed may give a better results in representation of the actual COP reduction.

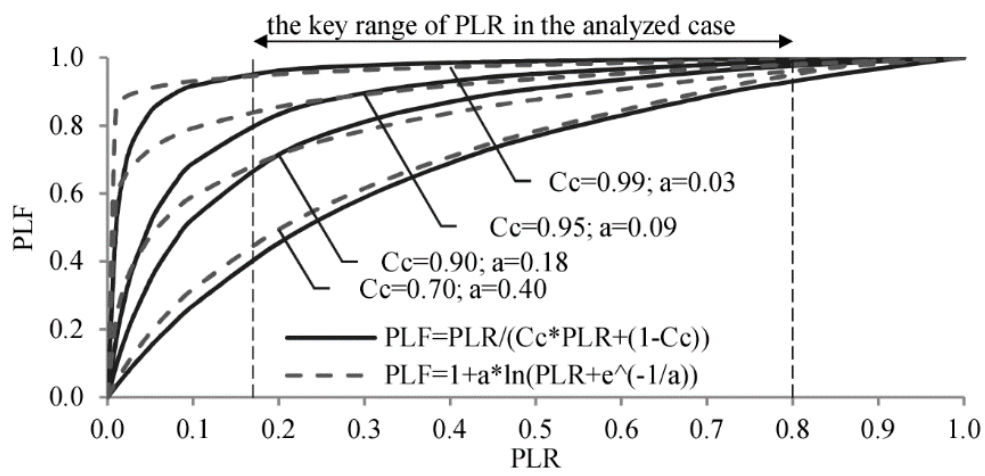

Fig. 3. The course of the functions for exemplary values of dependent parameters. 


\section{Conclusions}

Considering the degradation of the COP in the part-load operation is extremely important in the air-to-water heat pump energy efficiency analyzes. Currently, due to the lack of necessary input data, the value of the degradation coefficient $C c=0.9$ adopted in accordance with the EN 14825 standard is used. However, this value often does not reflect the actual degree of energy efficiency degradation of a unit. In addition, the analyzes performed and the literature clearly indicate that the energy consumption in the stand-by mode is not the only factor that reduces the COP of a air-to-water heat pump. Conducting tests covering all the relevant factors is therefore necessary.

In this paper, a quasi-dynamic model of estimating the part load factor based on the natural logarithm was proposed. The parameter in the function is defined as the operational reduction coefficient $a$, which covers all the factors that have an impact on the COP reduction in case of working in the part-load mode. The correct fitting of the function to the measurement data plot was shown and used to determine the dependent value. It has been proved that the determination of the parameter $a$ is possible using a relatively small number of data points without losing the accuracy. The further stage of this work will determine the minimum range and the number of measured data to properly determine the dependent value. This will allow to develop a device test method, providing full data needed to calculate the actual COP degradation in case of part-load operation conditions.

\section{References}

1. EN 14511:2013, Air conditioners, liquid chilling packages and heat pumps with electrically driven compressors for space heating and cooling (2013)

2. K. Piechurski, M. Szulgowska-Zgrzywa, J. Danielewicz, E3S Web of Conferences 17, 00072 (2017)

3. EN 14825:2013, Air conditioners, liquid chilling packages and heat pumps, with electrically driven compressors, for space heating and cooling — Testing and rating at part load conditions and calculation of seasonal performance (2013)

4. EN 15316-4-2, Heating systems in buildings - Method for calculation of system energy requirements and system efficiencies - Part 4-2: Space heating generation systems, heat pump systems (2008)

5. E. Fuentes, D. Waddicor, J. Salom, REHVA Journal, 45-49 (2016)

6. UNI 10963, Air conditioners, chillers and heat pumps. Determination of the part load performances

7. G. Mader, H. Madani, Energy and Buildings 81, 296-304 (2014)

8. J.M. Corberán, D. Donadello, I. Martínez-Galván, C. Montagud, International Journal of Refrigeration 36, 2251-2261 (2013) 